\title{
Barreiras para a prática de atividade física em adolescentes. Um estudo por grupos focais
}

Barriers related to physical activity practice in adolescents. A focus-group study.

\author{
Mariana Silva Santos 1,3 \\ Rogério César Fermino 1,3,4 \\ Rodrigo Siqueira Reis $1,2,3$ \\ Ana Carina Cassou \\ Ciro Romélio Rodriguez Añez 2,3
}

1. Universidade Federal do Paraná. Programa de Pós-Graduação em Educação Física. Paraná. Brasil.

2. Pontifícia Universidade Católica do Paraná.

Curso de Educação Física. Paraná. Brasil.

3. Pontifícia Universidade Católica do Paraná. Grupo de Pesquisa em Atividade Física e Qualidade de Vida. Paraná. Brasil.

\section{Bolsista CAPES}

Recebido em 01/07/09 Revisado em 09/09/09 Aprovado em 17/09/09
Resumo - O objetivo do estudo foi identificar as barreiras para a prática de atividade física em adolescentes. Foi utilizada a técnica de grupos focais em indivíduos com idades entre 15 e 18 anos (n=59; 50,8\% meninas) e constituídos oito grupos homogêneos de acordo com o gênero. A análise de conteúdo foi empregada para agrupar e classificar os relatos e em seguida, aplicou-se a estatística descritiva, utilizando-se a frequência relativa e absoluta dos relatos semelhantes com auxílio do software SPSS 11.0. As barreiras mais frequentes entre os adolescentes foram as que constituem as dimensões "psicológicas, cognitivas e emocionais" e "culturais e sociais". Para os meninos, as barreiras mais relatadas foram a preguiça, a falta de companhia e a falta de tempo. Para as meninas, por sua vez, a preguiça, a falta de companhia e a ocupação foram mais frequentes. Pode-se concluir que os adolescentes percebem barreiras de maneira distinta de acordo com o gênero, o que exige ações específicas para promoção da atividade física neste grupo.

Palavras-chave: Determinantes; Comportamento; Adolescência; Jovens; Exercício.

Abstract - The aim of study was to identify barriers to physical activity in adolescents. Focus group interviews were conducted with subjects aged 15 to 18 years ( $n=59,50.8 \%$ girls) and divided according to gender. Content analysis was used to classify the reports into specific dimensions. Descriptive statistics employing relative and absolute frequencies of similar reports was performed using the SPSS 11.0 software. The most frequent barriers among adolescents were those associated with "psychological, cognitive and emotional" and "cultural and social" dimensions. For boys, the most frequently reported barriers were "feeling lazy", "lack of company" and "lack of time". For girls, "feeling lazy", "lack of company" and "occupation" were the most common barriers. In conclusion, the perception of barriers by adolescents varies according to gender, a fact requiring specific actions for the promotion of physical activity in this group.

Key words: Determinants; Behavior; Adolescence; Youngsters; Exercise. 


\section{INTRODUÇÃO}

A inatividade física é, atualmente, considerada um dos maiores problemas de saúde pública e um dos principais fatores de risco para doenças crônicodegenerativas ${ }^{1}$. Estudos recentes demonstraram que os níveis de atividade física (AF) tendem a declinar com o avanço da idade ${ }^{2}$, em especial no período da adolescência ${ }^{2,3}$. No Brasil, estima-se que entre 39 e 93,5\% dos jovens são fisicamente inativos ${ }^{4}$. A participação em programas regulares de AF na adolescência proporciona melhora na saúde física e mental, além de possibilitar um estilo de vida saudável na idade adulta 5 .

A prática de AF pode ser influenciada de forma positiva ou negativa por diversos fatores. Quando esses influenciam de maneira negativa, são denominados barreiras. Sallis e Owen ${ }^{6}$ classificam esses determinantes em seis dimensões (demográficos e biológicos; psicológicos, cognitivos e emocionais; culturais e sociais; ambientais; características da AF e atributos comportamentais), o que demonstra a complexidade e diversidade dos aspectos que podem influenciar essa prática.

$\mathrm{Na}$ literatura internacional, diversas pesquisas têm objetivado identificar as barreiras para a prática de AF em adolescentes ${ }^{7-15}$. Sabe-se que elas podem variar de acordo com o estágio maturacional ${ }^{7}$ e a série escolar ${ }^{7,13}$. As principais barreiras relatadas são a falta de tempo $0^{7,10,11,14}$, falta de motivação ${ }^{7,10-12}$, falta de companhia ${ }^{7,10}$, possuir doença ou lesão ${ }^{7}$ preferência por atividades sedentárias ${ }^{10}$. No Brasil, existe uma carência de estudos que procuraram verificar essas barreiras. Nas únicas pesquisas identificadas no processo de revisão ${ }^{16,17}$, os motivos mais citados foram a falta de interesse pelo exercício, falta de conhecimento de como se exercitar ${ }^{16}$, falta de vontade e a obrigação com os estudos ${ }^{17}$.

$\mathrm{O}$ entendimento sobre os fatores que influenciam a prática de AF em adolescentes brasileiros ainda é limitado; não só, pela falta de estudos representativos da população, mas também pela carência de instrumentos que permitam a identificação desses fatores. Os estudos até agora realizados ${ }^{16,17}$ têm empregado instrumentos desenvolvidos em outros países, os quais podem não representar as características locais. Uma das maneiras de se compreender mais detalhadamente tais características é utilizar entrevistas, como aquelas conduzidas em grupos focais ${ }^{18}$.

De acordo com a revisão efetuada, parece não existir estudos na literatura que tenham utilizado a técnica de grupos focais com o intuito de identificar as barreiras para a AF em adolescentes no contexto brasileiro. O objetivo deste estudo foi realizar uma pesquisa exploratória para identificar as barreiras para a prática de $\mathrm{AF}$ em adolescentes.

\section{PROCEDIMENTOS METODOLÓGICOS}

\section{Participantes}

Participaram desta pesquisa 59 adolescentes (30 meninas - 50,8\%) estudantes do ensino médio, com idades entre 15 e 18 anos, de quatro instituições de ensino médio da cidade de Curitiba-PR. As escolas foram selecionadas intencionalmente, considerando sua região de localização (central e periferia), para representar diferentes realidades socioeconômicas e ambientais presentes na cidade. A seleção dos participantes ocorreu no início da aula de educação física e os adolescentes foram previamente comunicados. Com auxílio da lista de chamada do professor, foi realizado o sorteio dos alunos até que dois grupos homogêneos, quanto ao gênero e número de participantes, fossem constituídos em cada escola. Considerando as recusas de participação (5 indivíduos), obteve-se a amostra final utilizada na pesquisa.

Os indivíduos foram informados acerca dos procedimentos da pesquisa e aceitaram participar de maneira voluntária. Os pais e/ou responsáveis assinaram o Termo de Consentimento Livre e Esclarecido, autorizando os seus filhos a participarem. O estudo foi aprovado pelo Comitê de Ética em Pesquisa da Pontifícia Universidade Católica do Paraná, Brasil (processo n. ${ }^{\circ}$ 1076/2006).

\section{Elaboração dos Grupos Focais}

$\mathrm{O}$ número de participantes em cada grupo foi determinado de acordo com as recomendações de Stewart e Shamdasani ${ }^{18}$. Os autores sugerem que os grupos focais sejam homogêneos e constituídos por 12 integrantes. Grupos com número elevado de indivíduos dificultam o gerenciamento das entrevistas pelo moderador. Nesse estudo, optou-se por constituir grupos entre sete e oito indivíduos. Para garantir a homogeneidade de características dentro dos grupos, os participantes foram selecionados de acordo com o gênero. Desta maneira, foram formados oito grupos focais: quatro de meninos (três grupos com sete integrantes cada e um grupo com oito integrantes) e quatro de meninas (dois grupos com oito e dois com sete integrantes cada).

\section{Entrevistas em Grupos Focais}

As entrevistas seguiram um protocolo previamente preparado e testado em um estudo piloto. As infor- 
mações sobre o protocolo e outros detalhes estão disponíveis na literatura ${ }^{19}$. Foram tomados os devidos cuidados em relação aos aspectos éticos, local da aplicação, experiência do moderador, roteiro e duração da entrevista, bem como o registro das informações. Os grupos foram entrevistados em ambientes agradáveis, silenciosos e familiares para os participantes, para que esse não influenciasse a discussão.

A coordenação dos grupos foi realizada por um único pesquisador, previamente treinado para tal finalidade. As entrevistas seguiram um roteiro com questões abertas e tópicos vinculados aos fatores determinantes individuais, sociais e ambientais relacionados à prática de $\mathrm{AF}$. $\mathrm{O}$ roteiro foi dividido em quatro partes. A primeira consistiu na apresentação do mediador ao grupo, dos indivíduos entre si para promover a integração dos participantes e dos objetivos do estudo. A segunda parte caracterizouse pelo estímulo da discussão, quando os alunos foram incentivados a relatar o que gostavam de fazer durante a semana e finais de semana. $O$ mediador buscou aprofundar as respostas através de outras perguntas (o que? como? quando? onde? porque?, etc), evitando induzir as respostas esperadas. $\mathrm{Na}$ terceira parte, foram disponibilizadas diversas imagens, retratando comportamentos ativos e inativos de modo que os participantes deveriam selecionar figuras comuns ao seu dia-a-dia e posteriormente, explicar os motivos pela escolha. No quarto momento, dois temas foram apresentados. $\mathrm{O}$ primeiro tinha como objetivo saber se praticavam AF sozinhos ou acompanhados, bem como comentar suas respostas. $\mathrm{O}$ segundo referia-se a algo que os pais ou amigos faziam para auxiliar ou não a prática de AF. As questões foram elaboradas de maneira a não colocar os participantes em situações de constrangimento, tentado criar um ambiente favorável para a participação de todos perante o grupo.

A duração das entrevistas correspondeu, aproximadamente, ao tempo da aula de educação física (50 min), permitindo que todos os temas do roteiro fossem abordados sem dispersão dos participantes. As respostas e discussões foram registradas e gravadas por um auxiliar que não intervinha na discussão, obtendo-se, ao final de cada entrevista, um registro manuscrito das informações importantes e um registro em áudio na íntegra. A gravação foi iniciada após autorização dos participantes e finalizada após o encerramento da discussão e todos terem se retirado do local. Posteriormente, os arquivos foram transcritos na íntegra, atribuindo-se a cada participante um código (P1, P2, P3, etc.) para evitar a sua identificação.

\section{Análise dos Dados}

A análise de dados foi realizada de maneira qualiquantitativa. Na etapa qualitativa, as informações obtidas nos grupos focais foram analisadas com o emprego da análise de conteúdo. Os relatos com aspectos considerados negativos, ou barreiras, para a prática de AF foram identificados. Posteriormente, foram classificados em determinantes e dimensões, conforme a classificação de Sallis e Owen ${ }^{6}$. A Figura 1 apresenta um exemplo de classificação das barreiras identificadas.

$\mathrm{Na}$ etapa da análise quantitativa, após a classificação, aplicou-se a estatística descritiva, utilizando-se frequências relativas e absolutas dos relatos semelhantes, de acordo com o gênero. Todas as análises foram realizadas com auxílio do software SPSS 11.0.

\section{RESULTADOS}

Dentre os 176 relatos identificados nos grupos focais, foram incluídos aqueles que apresentavam frequência maior ou igual a dois. Desta maneira, foram considerados apenas 108 relatos, que resultaram em 17 barreiras. Os meninos identificaram 12 barreiras (50 relatos) que correspondem a 46,3\%

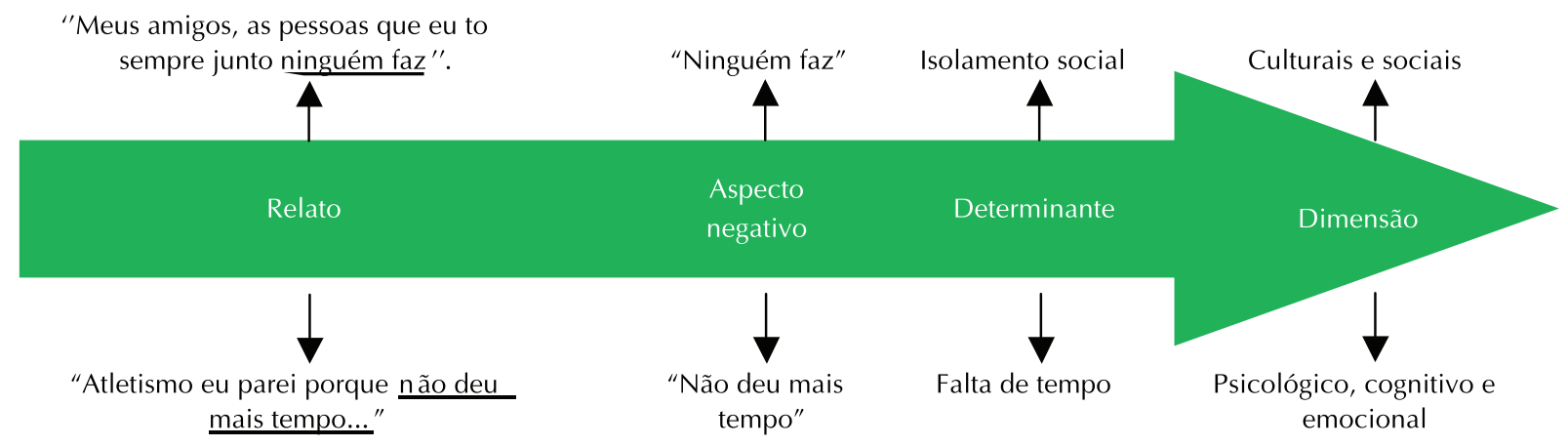

Figura 1. Classificação das barreiras para a prática de atividade física. 
Tabela 1. Frequências absolutas e relativas de barreiras para a prática de atividade física relatadas de acordo com o gênero

\begin{tabular}{|c|c|c|c|c|c|c|c|}
\hline \multicolumn{4}{|c|}{$\begin{array}{c}\text { Meninos } \\
(\mathrm{n}=29)\end{array}$} & \multicolumn{4}{|c|}{$\begin{array}{c}\text { Meninas } \\
(\mathrm{n}=30)\end{array}$} \\
\hline Determinante & $\mathrm{Fa}$ & $\%$ & Dimensão & Determinante & $\mathrm{Fa}$ & $\%$ & Dimensão \\
\hline Preguiça & 9 & 18 & Psi, Cog e Emo & Preguiça & 14 & 24,2 & Psi, Cog e Emo \\
\hline Falta de companhia & 9 & 18 & Cult e Soc & Falta de companhia & 8 & 13,8 & Cult e Soc \\
\hline Falta de tempo & 7 & 14 & Psi, Cog e Emo & Ocupação & 8 & 13,8 & Demo e Biol \\
\hline Baixa auto-eficácia & 6 & 12 & Psi, Cog e Emo & Falta de tempo & 4 & 6,9 & Psi, Cog e Emo \\
\hline Ocupação & 4 & 8 & Demo e Biol & Baixa auto-eficácia & 4 & 6,9 & Psi, Cog e Emo \\
\hline Falta de vontade & 3 & 6 & Psi, Cog e Emo & Condições climáticas & 3 & 5,2 & Amb \\
\hline Longe de casa & 2 & 4 & Amb & Não gosta de competição & 3 & 5,2 & Car AF \\
\hline Falta de incentivo financeiro & 2 & 4 & Psi, Cog e Emo & Ambiente fechado & 3 & 5,2 & Amb \\
\hline Amigos não chamam & 2 & 4 & Cult e Soc & Companhia atrapalha & 3 & 5,2 & Cult e Soc \\
\hline Falta de dinheiro & 2 & 4 & Demo e Biol & Falta de motivação & 2 & 3,4 & Psi, Cog e Emo \\
\hline Ambiente perigoso & 2 & 4 & $A m b$ & Falta de vontade & 2 & 3,4 & Psi, Cog e Emo \\
\hline \multirow[t]{2}{*}{ Acesso } & 2 & 4 & Amb & Falta de dinheiro & 2 & 3,4 & Demo e Biol \\
\hline & & & & Ambiente perigoso & 2 & 3,4 & Amb \\
\hline Total & 50 & 100 & & & 58 & 100 & \\
\hline
\end{tabular}

Fa: frequência absoluta; Psi, Cog e Emo: psicológicos, cognitivos e emocionais; Cult e Soc: culturais e sociais; Demo e Biol: demográficos e biológicos; Amb: ambientais; Car AF: características da atividade física.

dos relatos. Enquanto que as meninas identificaram 13 barreiras ( 58 relatos), que correspondem a 53,7\% dos relatos. Para os meninos, os motivos mais frequentes foram a preguiça, a falta de companhia $(9$ relatos cada - 18\%) e a falta de tempo (7 relatos 14\%). Para as meninas, as barreiras mais relatadas foram a preguiça (14 relatos $-24,2 \%)$, a falta de companhia e a ocupação (8 relatos cada - 13,8\%). As demais barreiras relatadas e as respectivas frequências e dimensões às quais pertencem são apresentadas na Tabela 1. Das principais barreiras identificadas em cada gênero, oito são comuns a ambos os gêneros (Figura 2).

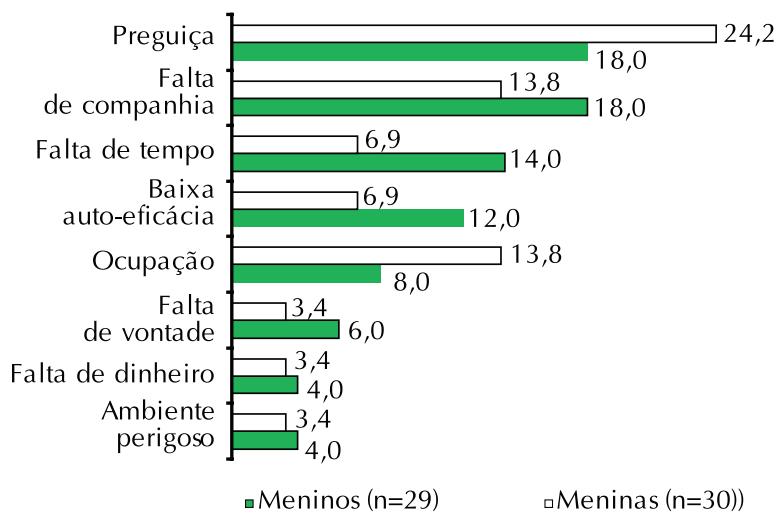

Figura 2. Frequência relativa (\%) das barreiras comuns a ambos os gêneros.

A Figura 3 apresenta a frequência das barreiras agrupadas por dimensões. Para ambos os gêneros, as dimensões "psicológica, cognitivas e emocionais" e "culturais e sociais" foram as mais relatadas.

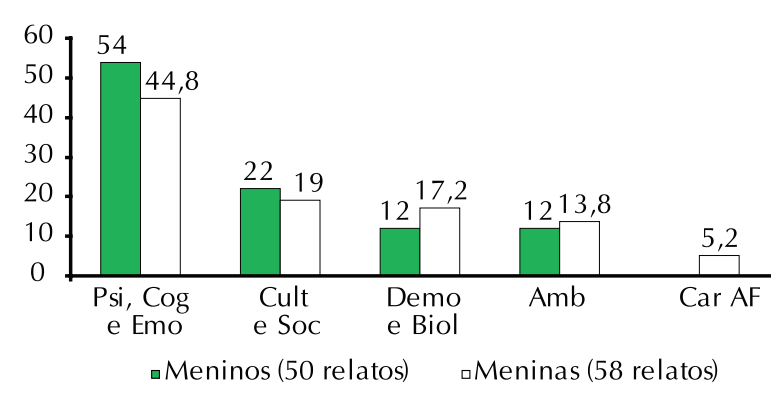

Figura 3. Percentual dos relatos classificados em dimensões de acordo com o gênero (as abreviações podem ser visualizadas na Tabela 1).

\section{DISCUSSÃO}

A presente pesquisa, de natureza exploratória e descritiva, teve como objetivo identificar as barreiras para a prática de $\mathrm{AF}$ em adolescentes, utilizando a técnica de grupos focais. Neste sentido, o estudo apresenta contribuições originais uma vez que são escassas pesquisas similares na literatura. A análise dos relatos permitiu observar algumas diferenças na percepção de barreiras quando considerado o gênero. Para ambos, os aspectos "psicológicos, cognitivos e emocionais" e "culturais e sociais" foram os mais relatados. Entretanto, as meninas relataram, com maior frequência, o motivo preguiça, enquanto, para os meninos, o motivo falta de companhia foi um dos mais importantes. A relevância em identificar as barreiras e sua respectiva dimensão reside na utilidade em direcionar estratégias de intervenção distintas para cada categoria? . Nesse sentido, os 
resultados proporcionados por estudos qualitativos podem ser úteis para auxiliar no desenvolvimento de comportamentos saudáveis ${ }^{12,20}$.

Durante a adolescência, é verificado um declínio dos níveis de $\mathrm{AF}^{2,3}$. Uma das possíveis explicações para esse fenômeno seria o número de barreiras que dificultam a participação em programas regulares de $A F^{10}$. No início da adolescência, são observadas diversas alterações comportamentais, socioculturais e maturacionais ${ }^{7,21}$ que poderiam explicar a menor participação nas atividades físicas. As barreiras são motivos percebidos pelos indivíduos que podem reduzir a chance de envolvimento na atividade ${ }^{7}$. De acordo com Allison et al. ${ }^{10}$, essas incluem fatores intrínsecos (características individuais, prioridades, etc.) e extrínsecos (falta de tempo, suporte familar ou de amigos).

Diversos estudos realizados com adolescentes mostraram que os meninos são mais ativos ${ }^{2,8,9,21-23}$. Devido ao fato da prática de AF ser um comportamento complexo, determinado por diversos fatores, é importante diferenciar os motivos que levam indivíduos de ambos os gêneros a optarem por estilos de vida ativos ou não. Não somente os aspectos biológicos tornam meninos e meninas diferentes, mas também as diversas razões no processo de criação, que também são distintos ${ }^{21,24}$, assim como fatores socioculturais ${ }^{22,24}$ poderiam explicar as diferenças na percepção das barreiras.

É consensual que aspectos demográfico-biológicos, psicológicos e socioculturais podem influenciar a heterogeneidade populacional nos hábitos de $\mathrm{AF}$ em adolescentes ${ }^{24}$. Diversos estudos, com diferentes metodologias, verificaram uma grande diversidade de barreiras para a AF. Os motivos mais citados são o fato de possuir doença ou lesão $0^{7,13}$, falta de tempo $0^{7,10,11,14,20}$, falta de motivação $0^{710,12,13}$, falta de companhia ${ }^{7,10,13}$, falta de locais ${ }^{11}$, características ambientais ${ }^{7}$ ou simplesmente a preferência por atividades sedentárias ${ }^{10}$ e outros interesses ${ }^{11,13,14}$. Contudo, no Brasil, são conhecidos apenas dois estudos que verificaram tais barreiras ${ }^{16,17}$. Ceschini e Figueira Jr. ${ }^{16}$ avaliaram 1738 adolescentes (58,5\% meninas, $16,1 \pm 1$ anos) de nível socioeconômico elevado e verificaram que os motivos mais relatados foram a falta de interesse pelo exercício e a falta de conhecimento de como se exercitar. Souza ${ }^{17}$, ao avaliar 2271 estudantes (55\% meninas, $16,2 \pm 1$ anos) de 29 escolas particulares, encontrou a falta de vontade e a obrigação com os estudos como as barreiras mais citadas. Apesar de amostrarem uma grande quantidade de indivíduos, em ambos os estudos, foi utilizado um questionário com perguntas fechadas. As diferenças quanto as barreiras apresentadas em estudos nacionais e internacionais poderiam ser explicadas pelas características físicas, sociais e ambientais de cada população, o que dificulta a comparação de resultados.

Em duas pesquisas recentes os autores recorreram a técnica de grupos focais para verificar as barreiras em meninos ${ }^{10}$ e meninas ${ }^{20}$ canadenses. $^{2}$ Os principais motivos relatados foram a falta de suporte dos amigos/família, falta de segurança, baixa prioridade para a AF, falta de tempo, falta de acesso e custo, baixa auto-eficácia, prioridade com os estudos, preferência em assistir televisão, utilizar computador e internet, jogar vídeo-game, conversar em chats e ao telefone. Em outro estudo com metodologia semelhante, $\mathrm{Vu}$ et al. ${ }^{15}$ verificaram que, para as meninas, a principal barreira era a presença de meninos, pois eles são injustos durante os jogos e atrapalham suas atividades.

\section{Barreiras de ordem psicológicas, cognitivas e emocionais}

Os fatores "psicológicos, cognitivos e emocionais", que apareceram como a primeira dimensão mais relatada por ambos os gêneros, são considerados como as causas mais comuns do comportamento inativo. As características dessa dimensão dizem respeito a valores, intenções, emoções, percepções e atitudes que ajudam a explicar os motivos pelos quais alguns indivíduos são fisicamente ativos ${ }^{6}$. No presente estudo, as barreiras mais relatadas dessa dimensão foram a preguiça, a falta de tempo e baixa autoeficácia.

As meninas relataram, com maior frequência, o motivo "preguiça", o que pode ser explicado pelo fato de optarem, por questões socioculturais, por atividades de lazer inativas ${ }^{21,24}$. Desde idades muito jovens, as meninas são direcionadas para o cuidado com a família enquanto os meninos são orientados a atividades laborais e de intensidade mais vigorosa ${ }^{24}$. Para os meninos, a falta de tempo também foi um motivo relevante, o que pode, em parte, ser atribuído a maior responsabilidade e tempo empregado em horas de estudo ${ }^{21}$. Nessa idade, os adolescentes se encontram em um estágio acadêmico em que existe uma intensificação na preparação para o processo seletivo de ingresso à universidade ${ }^{17}$. Para Dwyer et al ${ }^{20}, \mathrm{o}$ trabalho fora de casa, o envolvimento com tecnologias, além das lições de casa, também contribuíram para que esse motivo fosse um dos mais relatados. No presente estudo, a ocupação foi bastante relevante, em especial para as meninas. Apesar de não se enquadrar nas determinantes "psicológicos, cognitivos e emocionais" e sim como aspectos "demográficos e biológicos", para elas, essa barreira pode estar associada à necessidade de dedicar maior tempo a tarefas 
domésticas, além dos estudos. Estes resultados já haviam sido encontrados em adolescentes brasileiros ${ }^{21}$. No Brasil, estima-se que as mulheres dispendem, aproximadamente, três vezes mais tempo em afazeres domésticos que os homens (27,2 versus 10,7 hrs/semana $)^{25}$. Por essa razão, o acúmulo de tarefas poderia diminuir a disponibilidade de tempo e a disposição para outras atividades. Estas questões socioculturais impõem mudanças comportamentais que interferem na decisão de praticar AF e desenvolver outros hábitos saudáveis ${ }^{21}$. Para Tergerson e King ${ }^{11}$, uma solução coerente para a falta de tempo seria o engajamento efetivo nas aulas de educação física, pois não requer tempo adicional fora do horário escolar.

A baixa autoeficácia tem sido, frequentemente, relatada em estudos com adolescentes ${ }^{8-10}$. Ela é descrita na literatura como um fator que influencia o poder de determinar o grau de persistência do comportamento frente às dificuldades e o sucesso no desempenho, refletindo não somente na $\mathrm{AF}$ realizada no passado, mas também acrescentando influência na atividade futura $^{26}$. A percepção de baixa autoeficácia faz com que os adolescentes desistam de realizar determinadas atividades, uma vez que passa a não acreditar em sua competência ou capacidade de realizar a tarefa. Kohl $111 \mathrm{e} \mathrm{Hobbs}^{27}$ sugerem a autoeficácia não simplesmente como um fator relacionado a $\mathrm{AF}$, e sim como preditor do comportamento fisicamente ativo. Em outros estudos de revisão, a autoeficácia foi associada ao comportamento fisicamente ativo de adolescentes ${ }^{8,9}$.

\section{Barreiras de ordem culturais e sociais}

Os fatores "sociais e culturais", que apareceram como a segunda dimensão mais relatada por ambos os gêneros, referem-se, principalmente, ao isolamento social (falta de companhia) e suporte social dos amigos e da família ${ }^{6}$. A barreira falta de companhia foi relatada pelos jovens de ambos os gêneros como um dos principais motivos para não praticarem AF. Apesar das diferenças metodológicas, Gonçalves et al. ${ }^{21}$ verificaram associação entre a possibilidade de encontrar amigos e a inatividade física. Foi verificada uma relação inversa entre a prevalência de inatividade física e o número de dias na semana em que os adolescentes se encontram com os amigos ${ }^{21}$. Outros motivos poderiam explicar a relação entre a falta de companhia e a AF. Em adolescentes brasileiros, Hallal et al..$^{23}$ verificaram associação positiva entre a inatividade física e tempo diário assistindo televisão. Uma hipótese é que tais atividades podem ser desenvolvidas de maneira individual e que jovens sem companhia para a prática de AF recorreriam a atividades de lazer inativas. Entretanto, são necessários estudos para melhor elucidar esta suposição. Existem evidências que jovens fisicamente ativos tem amigos igualmente ativos ${ }^{24}$. Outros autores têm encontrado uma forte associação entre a AF e o suporte social da família, amigos e outras pessoas importantes para os jovens $s^{6,8}$. A força dessa associação contribui para que o suporte social seja, frequentemente, abordado na literatura como uma estratégia de intervenção efetiva na promoção de um estilo de vida ativo ${ }^{26}$. Esses resultados reforçam os achados de Van de Horst et al. ${ }^{9}$, nos quais o suporte social da família e dos amigos esteve associado a AF.

Um aspecto a ser considerado neste estudo refere-se ao número de participantes. A amostra foi constituída por 59 adolescentes, sendo, portanto, não representativa da população. A metodologia empregada teve como intuito compreender a opinião dos sujeitos, não sendo possível a inclusão de um número elevado de pessoas e grupos, uma vez que o tempo das entrevistas e a transcrição das gravações são elevados. Apesar dessa caracteristica metodológica, a técnica de grupos focais possibilita a identificação real de informações dos indivíduos, proporcionando discussões entre os participantes que estimulam a percepção de diversas características comportamentais que não são identificadas quando se utiliza questionários. Ainda que nesse estudo tenha sido realizado um esforço para identificar as barreiras para a prática de AF em adolescentes, alguns cuidados devem ser tomados ao extrapolar os resultados encontrados. A região Sul do país apresenta elevados índices sociais e devido à grande extensão territorial brasileira, outras regiões podem não representar as mesmas características. Os resultados desse estudo podem subsidiar a elaboracão de instrumentos mais objetivos, como questionários e escalas, que avaliem as barreiras para a $\mathrm{AF}$ de adolescentes em contextos similares. Sugere-se que outras pesquisas, com amostras diversas, sejam conduzidas com o intuito de verificar a consistência dos resultados em outras comunidades.

\section{CONCLUSÃO}

Conclui-se que os adolescentes de ambos os gêneros percebem as barreiras para a AF de maneira distinta, sendo os fatores "psicológicos, cognitivos e emocionais" e "culturais e socias" mais relevantes. Para os meninos, as barreiras mais relatadas foram a preguiça, a falta de companhia e a falta de tempo. Para as meninas, as mais importantes foram a preguiça, a falta de companhia e a ocupação. De acordo com os resultados, pode-se inferir que as barreiras identifica- 
das são passíveis de modificação com programas de intervenção, o que não seria possível se essas fossem de ordem ambiental. A identificação das barreiras e fatores determinantes para o envolvimento em AFé uma importante estratégia que possibilita obter informações de grande utilidade para o desenvolvimento de programas de intervenção e promoção da saúde.

\section{REFERÊNCIAS BIBLIOGRÁFICAS}

1. World Health Organization. World Health Report 2002. Reducing risks, promoting healthy life. Geneva: WHO, 2002; Available from: <http://www.who.int/ whr/2002/en/> [2008 May 20).

2. Caspersen CJ, Pereira MA, Curran KM. Changes in physical activity patterns in the United States, by sex and cross-sectional age. Med Sci Sports Exerc 2000;32(9):1601-1609.

3. Allison KR, Adlaf EM, Dwyer JJM, Lysy DC, Irving HM. The decline in physical activity among adolescent students. A cross-national comparison. Can J Public Health 2007;98(2):97-100.

4. Tassitano RM, Bezerra J, Tenório MCM, Colares V, Barros MVG, Hallal PC. Atividade física em adolescentes brasileiros: uma revisão sistemática. Rev Bras Cineantropom. Desempenho Hum 2007;9(1):55-60.

5. Hallal PC, Victora CG, Azevedo MR, Wells JC. Adolescent physical activity and health: a systematic review. Sports Med 2006;36(12):1019-1030.

6. Sallis JF, Owen N. Physical Activity \& Behavioral Medicine. California: SAGE Publications. Behavioral Medicine and Health Psychology Series. 1999.

7. Sherar LB, Gyurcsik NC, Humbert ML, Dyck RF, Fowler-Kerry S, Baxter-Jones ADG. Activity and barriers in girls (8-16 yr) based on grade and maturity status. Med Sci Sports Exerc 2009;41(1):87-95.

8. Sallis JF, Prochaska JJ, Taylor WC. A review of correlates of physical activity of children and adolescents. Med Sci Sports Exerc 2000;32(5):963-975.

9. Van Der Horst K, Paw MJ, Twisk JW, Van Mechelen W. A brief review on correlates of physical activity and sedentariness in youth. Med Sci Sports Exerc 2007;39(8):1241-1250.

10. Allison KR, Dwyer JJ, Goldenberg E, Fein A, Yoshida KK, Boutilier M. Male adolescents' reasons for participating in physical activity, barriers to participation, and suggestions for increasing participation. Adolescence 2005;40(157):155-170.

11. Tergerson JL, King KA. Do perceived cues, benefits, and barriers to physical activity differ between male and female adolescents. J School Health 2002;79(9):374-380.

12. Gordon-Larsen P, Griffiths P, Bentley ME, Ward DS, Kelsey K, Shields K, et al. Barriers to physical activity qualitative data on caregiver-daughter perceptions and practices. Am J Prev Med 2004;27(3):218-223.

13. Gyurcsik NC, Spink KS, Bray SR, Chad K, Kwan M. An ecologically based examination of barriers to physical activity in students from grade seven through firstyear university. J Adolesc Health 2006;38:704-711.

14. Kimm SY, Glynn NW, McMahon RP, Voorhees CC, Striegel-Moore RH, Daniels SR. Self-perceived barriers to activity participation among sedentary adolescent girls. Med Sci Sports Exerc 2006;38(3):534-540.

15. Vu MB, Murrie D, Gonzalez V, Jobe JB. Listening to girls and boys talk about girls' physical activity beha- viors. Health Educ Behav 2006;33(1):81-96.

16. Ceschini FL, Figueira Jr. A. Barreiras e determinantes para a prática de atividade física em adolescentes. $R$ bras Ci e Mov 2007;15(1):29-36.

17. Souza GS. Determinantes da atividade física e estágios de mudança de comportamento em adolescentes [Dissertação de Mestrado - Programa de Pós-Graduação em Educação Física]. Florianópolis: Centro de Desportos, Universidade Federal de Santa Catarina. 2003.

18. Stewart DW, Shamdasani PN. Focus groups: Theory and practice. California: Sage: Newbury Park; 1990.

19. Santos MS, Reis RS, Rodriguez-Añez CR, Fermino RC. Desenvolvimento de um instrumento para avaliar barreiras para a prática de atividade física em adolescentes. Rev Bras Ativ Fís Saúde 2009;14(2):76-85.

20. Dwyer JJ, Allison KR, Goldenberg ER, Fein AJ, Yoshida KK, Boutilier MA. Adolescent girls' perceived barriers to participation in physical activity. Adolescence 2006;41(161):75-89.

21. Gonçalves H, Hallal PC, Amorim TC, Araújo CLP, Menezes AMB. Sociocultural factors and physical activity level in early adolescence. Pan Am J Public Health 2007;22(4):246-253.

22. Teixeira e Seabra AF, Maia JA, Mendonca DM, Thomis M, Caspersen CJ, Fulton JE. Age and sex differences in physical activity of Portuguese adolescents. Med Sci Sports Exerc 2008;40(1):65-70.

23. Hallal PC, Bertoldi AD, Gonçalves H, Victora CG. Prevalência de sedentarismo e fatores associados em adolescentes de 10-12 anos de idade. Cad Saúde Pública 2006; 22(6):1277-1287.

24. Seabra A, Mendonça DM, Thomis MA, Anjos LA, Maia JAR. Determinantes biológicos e sócio-culturais associados à prática de atividade física de adolescentes. Cad Saúde Pública 2008;24(4):721-736

25. Pinheiro L, Fontoura NO, Querino AC, Bonetti A Rosa W. Retrato das desigualdades de gênero e raça; Available from: <http://www.ipea.gov.br/default.jsp > [2008 Dez 28].

26. Mota J, Sallis JF. Actividade Física e Saúde: factores de influência da atividade física nas crianças e nos adolescentes: Ed Campo das letras; 2002.

27. Kohl $111 \mathrm{HW}$, Hobbs KE. Development of physical activity behaviors among children and adolescents. Pediatrics 1998;101(3 Pt 2):549-554.

\footnotetext{
Endereço para correspondência

Mariana Silva Santos

Pontifícia Universidade Católica do Paraná - PUCPR

Centro de Ciências Biológicas e da Saúde, CCBS

- Curso de Educação Física

Grupo de Pesquisa em Atividade Física e Quali-

dade de Vida - GPAQ

Rua Imaculada Conceição, 1155 - Prado Velho CEP 80215-901

Curitiba - Paraná - Brasil

E-mail: marianass@pop.com.br
} 